\title{
Evaluación de las Diferencias en la Motivación de Estudiantes de Educación Física en Secundaria, según las Estrategias del Profesor para Mantener la Disciplina
}

\author{
Motivational Profiles in Physical Education. Differences according \\ to Teachers Strategies to Maintain Discipline
}

\author{
Antonio Granero-Gallegos*, ${ }^{*}$, Manuel Gómez-López ${ }^{b}$, Antonio Baena-Extremera ${ }^{b}$, \\ Clara Bracho-Amador ${ }^{c} \&$ Francisco Javier Pérez-Quero ${ }^{c}$ \\ ${ }^{a}$ Consejería de Educación, Cultura y Deporte, Junta de Andalucía, Andalucía, España, \\ ${ }^{b}$ Universidad de Murcia, Murcia, España \& ${ }^{c}$ Universidad de Huelva, Andalucía, España
}

\begin{abstract}
Resumen
El objetivo del estudio fue analizar las diferencias motivacionales del alumnado en las clases de Educación Física, según las estrategias de disciplina del profesor y analizar las relaciones de los conglomerados con el clima motivacional, orientaciones de meta y satisfacción. Participaron 2002 alumnos (970 varones y 1032 mujeres) de educación secundaria de entre 12-19 años. Como instrumentos se utilizaron la versión española de: Strategies to Sustain Discipline Scale, Learning and Performance Orientations in Physical Education Classes Questionnaire, Perception of Success Questionnaire y Sport Satisfaction Instrument. Se realizó un análisis de conglomerados que reveló tres perfiles de disciplina: uno con énfasis en estrategias autodeterminadas para mantener la disciplina en clase y dos con énfasis en estrategias no autodeterminadas Se hallaron el $n^{\circ}$ de horas semanales que practican ejercicio físico en su tiempo libre en función del perfil. El conglomerado de estrategias autodeterminadas está compuesto fundamentalmente por mujeres y los no autodeterminados por varones. En el de estrategias autodeterminadas se ubica el mayor número de estudiantes que practican dos o más horas semanales de ejercicio físico. Para mejorar el ambiente de clase y reducir las conductas de indisciplina, se deberían utilizar estrategias de disciplina fundamentadas en razones intrínsecas, de preocupación y responsabilidad.

Palabras clave: Conglomerados, educación secundaria, sexo, práctica deportiva.
\end{abstract}

\begin{abstract}
The aim of the study was to detect the motivational profiles of high school students of Physical Education (PE) according to the teacher's discipline strategies and to analyze the relationships between the clusters and the motivational atmosphere, goal guidelines and satisfaction with lessons. A sample of 2002 students ( 970 male students and 1032 female students) was used. The instruments used were the Spanish versions of the scales: Strategies to Sustain Discipline Scale, Learning and Performance Orientations in Physical Education Classes Questionnaire, Perception of Success Questionnaire and Sport Satisfaction Instrument. A cluster analysis was applied revealing three motivational profiles, one of them with emphasis on self-determined strategies to maintain discipline in class and the other two profiles with emphasis on non-self- determined-strategies. Profiles were determined according to the number of hours of exercise practiced in a week. The self-determined strategies cluster consists mainly of women, and the non- self-determined clusters were composed of men. In the self-determined strategies cluster, we can find the largest number of students who practice two or more hours of physical exercise per week in their free time. To improve the classroom environment and reduce indiscipline behaviors, the teacher should use discipline strategies founded on intrinsic reasons for concern and responsibility.

Keywords: Cluster, secondary education, gender, sports.
\end{abstract}

La preocupación por el control y la disciplina en el contexto de enseñanza-aprendizaje ha crecido en los últi-

\footnotetext{
${ }^{*}$ Dirección postal: Facultad de Ciencias del Deporte, Universidad de Murcia, Calle Argentina, s/n, Santiago de la Ribera, Murcia, España, 30720. E-mail: abaenaextrem@um.es
}

mos años en el entorno educativo debido, sobre todo, a la aparición de conductas indisciplinadas (Gutiérrez, López, \& Ruiz, 2009; Gutiérrez, Ruiz, \& López, 2010). Bekiari, Kokaridas, y Sakellariou (2006) afirman que uno de los indicadores más importantes del éxito en la enseñanza es una clase disciplinada. Aquellos docentes que controlen el tiempo y el comportamiento del alumnado en clase podrán 
aumentar el tiempo de aprendizaje (Kulinna, Cothran, \& Regualos, 2003); en esta línea, uno de los aspectos que junto al control y la disciplina resulta fundamental para garantizar un ambiente de clase capaz de potenciar el aprendizaje, es la motivación del alumno durante el desarrollo de la clase. Rutten, Boen, y Seghers (2012) afirman que esta motivación no depende únicamente de la percepción que los estudiantes puedan tener de la asignatura, ni de los agentes psicosociales que influyen en ellos en la etapa de socialización, sino también del ambiente de clase. Estudios como el realizado por Cervelló, Jiménez, Del Villar, Ramos, y Santos-Rosa (2004) han demostrado la relación entre disciplina y motivación. Los resultados de la investigación realizada por Papaioannou (1998) muestran una asociación entre las conductas de disciplina en clase y la motivación de los alumnos por aprender, al igual que entre la indisciplina y la desmotivación escolar.

Siguiendo con el análisis de la motivación, son numerosas las investigaciones que han analizado la motivación en las clases de Educación Física (EF). Dentro de estas hay que resaltar aquellas que con el fin de proporcionar información relevante al alumno y posibilitar una enseñanza más individualizada que atienda a las necesidades motivacionales específicas de cada estudiante, han establecido perfiles motivacionales (Gómez-López, Granero-Gallegos, Baena-Extremera, \& Abraldes, 2013; Granero-Gallegos, Baena-Extremera, Pérez-Quero, Ortiz-Camacho, \& Bracho-Amador, 2012; Méndez-Giménez, Fernández-Río, Cecchini, \& González, 2013). Estos perfiles han permitido determinar unos patrones motivacionales en las muestras analizadas atendiendo a diferentes variables motivacionales. La revisión de estos estudios muestra que su constructo teórico se ha sustentado fundamentalmente en dos de las teorías más utilizadas actualmente en el campo de la psicología del deporte; por un lado, en la Teoría de las Metas de Logro (Nicholls, 1989) y, por otro, en la Teoría de la Autodeterminación (Deci \& Ryan, 1985, 2000; Ryan \& Deci, 2000). Este hecho, junto a la consideración de que la clase de EF es un contexto de logro, donde el alumno intenta demostrar su nivel de competencia y habilidad en el desarrollo de las clases y donde puede o no realizar de manera voluntaria las actividades que le ayuden a conseguir las metas de aprendizaje, ha supuesto que en la presente investigación se utilicen ambas teorías.

La teoría de las Metas de Logro se utiliza para conocer la orientación disposicional y el entorno de logro percibido por el alumnado a través de la descripción del clima motivacional (Nicholls, 1989). Por otro lado, la teoría de la Autodeterminación sugiere que la motivación se manifiesta a lo largo de un continuum en el que se establecen diferentes niveles de autodeterminación, en función de la voluntariedad con la que se realice las actividades. De este modo, de mayor a menor grado de autodeterminación, la conducta puede estar intrínsicamente motivada, extrínsecamente motivada o amotivada. Basándose en esta teoría, Papaioannou (1998) estableció diferentes estrategias que el docente puede utilizar con el fin de mantener la disciplina en las clases de EF. De este modo, el docente puede hacer énfasis en razones de preocupación y responsabilidad, en razones intrínsecas e identificadas, en razones introyectadas y de indiferencia. Spray y Wang (2001), utilizando ambas teorías, hallaron que los estudiantes con una alta orientación a la tarea y al ego y una relación positiva con la motivación intrínseca eran los más disciplinados en clase. Posteriormente Moreno et al. (2008) hallaron una relación positiva entre la orientación a la tarea y las estrategias de preocupación y responsabilidad e intrínsecas, y una relación negativa con la indiferencia mostrada por los docentes con respecto a la disciplina. Asimismo, también encontraron relaciones positivas entre la orientación al ego y la indiferencia del docente, y negativa con las estrategias de preocupación y responsabilidad e intrínsecas. Estos resultados fueron similares a los hallados con anterioridad por Moreno, Alonso, Martínez-Galindo, y Cervelló (2005) y Papaioannou (1998), donde se descubrió la relación existente entre la orientación a la tarea y el énfasis del docente en razones intrínsecas para mantener la disciplina y la orientación al ego y la regulación de la conducta del alumno mediante castigos y recompensas. Es decir, los tipos de motivación más autodeterminados se relacionan positiva y significativamente con las estrategias de preocupación, responsabilidad e intrínsecas que favorecen la disciplina y negativamente con las estrategias que promueven conductas menos disciplinadas como la indiferencia (Moreno, Hellín, Hellín, \& Cervelló, 2006). Papaioannou (1998) reflejó que los docentes que promueven una fuerte orientación a la tarea en sus alumnos y ayudan a adoptar mayores razones auto-determinadas para ser disciplinados fomentarán un ambiente de clase positivo que favorezca el aprendizaje. Por tanto, los docentes deben dirigir al alumno a través de estrategias de disciplina de preocupación y responsabilidad para lograr una orientación a la tarea y una motivación autodeterminada (Moreno et al., 2008).

Por otro lado, los estudios reflejan que la orientación a la tarea y la percepción de un clima motivacional al aprendizaje se relaciona de manera positiva con las razones intrínsecas de disciplina en clase (Cervelló et al., 2004; Cervelló et al., 2002; Moreno et al., 2005; Papaioannou, 1998). Asimismo, Cervelló et al. (2004), Papaioannou (1998), y Spray (2002), demostraron que la percepción de un clima motivacional al rendimiento tiene una relación positiva con estrategias docentes basadas en razones externas. Gutiérrez et al. (2010) hallaron que los predictores más importantes para conseguir una motivación intrínseca son la percepción de un clima al aprendizaje y la percepción de un énfasis del docente en razones intrínsecas para mantener la disciplina en clase. Así, si el docente pretende lograr comportamientos disciplinados en clase debe, por un lado, promover un clima motivacional al aprendizaje que refuerce la orientación a la tarea del alumno y, por otro, emplear estrategias que fomenten la autodeterminación (Spray \& Wang, 2001). 
Respecto al efecto del género en los factores objeto de estudio, los resultados hallados por Moreno et al. (2006) muestran que los varones presentan mayores niveles de orientación al ego, motivación extrínseca y percepción de estrategias de disciplina reguladas intrínsecamente, así como de indiferencia por el docente. En cambio, las mujeres están más motivadas intrínsecamente y perciben mejor las estrategias de disciplina en la preocupación responsabilidad. De este modo se ha comprobado que los varones perciben en mayor medida el énfasis del docente en estrategias que promueven la indisciplina, mientras que las mujeres perciben más las estrategias que promueven la disciplina (Alonso, Martínez-Galindo, Moreno, \& Cervelló, 2005; Martínez, Alonso, Moreno, \& Cervelló, 2005a; Moreno et al., 2006).

En cuanto a la variable práctica de ejercicio físico fuera del horario escolar, los resultados hallados por Martínez et al. (2005a) mostraron que las estrategias que promueven conductas de disciplina son mejor percibidas por los que practicaban; mientras que la indiferencia del docente por mantener la disciplina en clase es mejor percibida por los que no practican. Posteriormente Moreno et al. (2006) hallaron diferencias significativas en los que practicaban con los tipos de motivación intrínseca y extrínseca, excepto en la amotivación, y con las estrategias de disciplina basadas en la motivación intrínseca. Asimismo, estos autores también encontraron diferencias significativas en aquellos alumnos no practicantes con la amotivación y con la indiferencia.

El objetivo principal del estudio fue analizar las diferencias motivacionales de los alumnos en las clases de EF según las estrategias de disciplina del docente. Los objetivos secundarios fueron: analizar las relaciones existentes entre los conglomerados y el clima motivacional percibido, las orientaciones de meta y la satisfacción con las clases de EF; y hallar las diferencias en dichos conglomerados atendiendo al sexo y el número de horas de ejercicio físico que realiza el alumno en su tiempo libre. Como primera hipótesis, se estiman tres perfiles de disciplina: uno con énfasis en estrategias autodeterminadas para mantener la disciplina en clase y caracterizado por la percepción de un clima motivacional de aprendizaje, una orientación a la tarea y sentimiento de diversión y placer con la práctica de actividades físicas y deportivas en las clases de EF; otro perfil sería el que hace énfasis en estrategias no autodeterminadas; caracterizado por la percepción de un clima motivacional de rendimiento, orientación al ego y sensación de aburrimiento con las clases de EF; y un tercer perfil basado también en estrategias no autodeterminadas, caracterizado por la indiferencia por parte del docente. Como segunda hipótesis, se cree que los conglomerados basados en estrategias autodeterminadas que promuevan la disciplina estarán formados sobre todo por mujeres y por los más activos en su tiempo libre; mientras que aquellos conglomerados no autodeterminados, que no favorecen las conductas de disciplina, estarán compuestos sobre todo por varones y por los que menos activos físicamente.

\section{Método}

\section{Participantes}

Para la selección de la muestra se utilizó un muestreo aleatorio por conglomerados, entendiendo estos como las diferentes clases de EF de los distintos centros a los que se accedió. Participaron en total 2002 alumnos (970 varones $=48.5 \% ; 1032$ mujeres $=51.5 \%$ ) de 17 centros andaluces de Educación Secundaria de las provincias de Almería, Córdoba, Granada, Jaén y Sevilla. El rango de edad estuvo comprendido entre 12 y 19 años $(M=14.99$; $S D=1.43)$, siendo la edad media de los varones 15.06 $(S D=1.43)$, y la de las mujeres $14.93(\mathrm{~S} D=1.43)$.En este estudio solo participaron estudiantes de secundaria que en la primavera del curso 2011/2012 respondieron a un cuestionario compuesto con los instrumentos que se exponen a continuación.

\section{Instrumentos}

Escala de Estrategias del Profesor para Mantener la Disciplina en Clase (SDSS). Se utilizó la versión española (Gutiérrez et al., 2009) del original Strategies to Sustain Discipline Scale (Papaioannou, 1998). Consta de 27 ítems que miden la percepción de los estudiantes de las estrategias utilizadas por los profesores para mantener la disciplina en las clases de EF. Inicialmente compuesto por cuatro factores (Papaioannou, 1998), en su validación española en estudiantes secundaria conservó tres dimensiones: énfasis del profesor sobre razones intrínsecas para mantener la disciplina (16 ítems, e.g. "Atrae nuestra atención y hace la clases más interesante"), énfasis del profesor sobre razones introyectadas para mantener la disciplina (4 ítems, e.g. "Nos recuerda que sólo los disciplinados son considerados buenos alumnos") e indiferencia del profesor para mantener la disciplina (5 ítems, e.g. "No hace nada por mantener la disciplina"), siendo eliminados dos ítems de la escala original (Gutiérrez et al., 2009): "Nos recuerda que ser disciplinado es la norma de la clase" y "Nos recuerda que hemos asumido ser disciplinados". En las instrucciones se pide a los alumnos que indiquen el grado de acuerdo o desacuerdo con los ítems, teniendo en cuenta la actuación del profesor de EF. Las respuestas se recogieron en una escala de ítems potilómicos de cinco puntos que oscila entre 1 (totalmente en desacuerdo) y 5 (totalmente de acuerdo). El análisis de consistencia interna resultó satisfactorio: énfasis razones intrínsecas, alfa de Cronbach $(\alpha)=.94$; énfasis razones introyectadas, $\alpha=$ .73; indiferencia, $\alpha=.71$.

Cuestionario de Orientación al Aprendizaje y al Rendimiento en las Clases de EF (LAPOPECQ). Se utilizó la versión española (Cervelló et al., 2002) del original Learning and Performance Orientations in Physical Education Classes Questionnaire (Papaioannou, 1998). Esta escala mide la percepción de los estudiantes del clima motivacional en las clases de Educación Física. Está compuesta por 27 ítems y posee dos dimensiones: percepción del clima motivacional al aprendizaje (clima 
Granero-Gallegos, A., Gómez-López,M., Baena-Extremera, A., Bracho-Amador, C. \& Pérez-Quero, F. J. (2015). Evaluación de las Diferencias en la Motivación de Estudiantes de Educación Física en Secundaria, según las Estrategias del Profesor para Mantener la Disciplina.

tarea; 13 ítems, e.g. "Me siento muy satisfecho cuando aprendo nuevas habilidades o juegos") y percepción del clima motivacional al rendimiento (clima ego; 14 ítems, e.g. "Los alumnos intentan conseguir recompensas sobresaliendo sobre los otros compañeros"). En las instrucciones se pide a los alumnos que indiquen el grado de acuerdo o desacuerdo con los ítems. Las respuestas se recogieron en una escala de ítems politómicos de diez puntos que oscila entre 0 (totalmente en desacuerdo) y 10 (totalmente de acuerdo). El análisis de consistencia interna de este estudio fue: clima al aprendizaje, $\alpha=.91$; clima al rendimiento, $\alpha=.88$.

Cuestionario de Percepción de Éxito (POSQ). Se utilizó la versión española adaptada a la EF (Martínez, Alonso, \& Moreno, 2006) del original Perception of Success Questionnaire (Roberts, Treasure, \& Balagué, 1998). Consta de 12 ítems para medir las orientaciones de meta disposicionales del alumnado en las clases de Educación Física, mediante dos dimensiones que miden la orientación hacia la tarea (6 ítems, e.g. "Cuando rindo a mi mejor nivel de habilidad") y la orientación hacia el ego (6 ítems, e.g. "Cuando mi actuación supera la del resto de alumnos"). En las instrucciones se pide a los alumnos que indiquen el grado de acuerdo o desacuerdo con los ítems. Las respuestas se recogieron en una escala de ítems politómicos de cinco puntos que oscila entre 1 (muy en desacuerdo) y 5 (muy de acuerdo). El análisis de consistencia interna fue: orientación tarea, $\alpha=.73$; orientación ego, $\alpha=.83$.

Escala de Satisfacción (SSI). Se utilizó la versión española adaptada a la EF (SSI-EF; Baena-Extremera, Granero-Gallegos, Bracho-Amador, \& Pérez-Quero, 2012) del original Sport Satisfaction Instrument (Duda \& Nicholls, 1992). Consta de 8 ítems para medir la satisfacción en la EF, mediante dos subescalas que miden satisfacción/ diversión (5 ítems, e.g. "Normalmente me divierto en las clases de Educación Física") y aburrimiento (3 ítems, e.g. "En las clases de Educación Física, normalmente me aburro") en las clases de EF. En las instrucciones se pide a los alumnos que indiquen el grado de acuerdo o desacuerdo con los ítems. Las respuestas se recogieron en una escala de ítems politómicos de cinco puntos que oscila entre 1 (muy en desacuerdo) y 5 (muy de acuerdo). El análisis de consistencia interna hallado fue: satisfacción/diversión, $\alpha$ $=.92$ y aburrimiento, $\alpha=.79$.

\section{Procedimiento}

Se obtuvo permiso para realizar la investigación por parte de los órganos competentes, tanto de los centros educativos de secundaria como universitarios. Se informó, en detalle, a padres/tutores y adolescentes acerca del protocolo y objeto del estudio. La firma del consentimiento informado por parte de ambos fue requisito indispensable para poder participar, en base a la Declaración de Helsinki (2008). No hubo ningún tipo de discriminación por razones raciales, sociales, de sexo o religión. Los instrumentos para medir las diferentes variables se administraron en el aula por los propios investigadores y sin la presencia del docente. Todos los participantes fueron informados del objetivo de estudio, voluntariedad y confidencialidad de las respuestas y manejo de datos y que no había respuestas correctas o incorrectas. Cada participante tuvo 20-30 minutos para completar los cuestionarios y se les pidió que contestaran con la máxima sinceridad y honestidad.

\section{Análisis Estadístico}

Se calcularon los estadísticos descriptivos, correlación entre las subescalas y consistencia interna de cada subescala. Seguidamente se realizaron dos análisis de conglomerados, atendiendo las sugerencias de Hair, Anderson, Tatham, y Black (1999). Se dividieron aleatoriamente los participantes por provincias andaluzas. Se realizó un análisis jerárquico exploratorio con un grupo mediante el método de Ward y se verificaron estos resultados obteniendo los perfiles motivacionales del otro grupo a través del método k-medias. Finalmente, se realizó un último análisis con toda la muestra a través del método de Ward. Se realizó un análisis multivariante (MANOVA) en el que las dimensiones del clima motivacional, orientaciones de meta y satisfacción en las clases de EF actuaron como variables dependientes y los conglomerados formados atendiendo a las dimensiones de la Escala de Estrategias del Profesor para Mantener la Disciplina en Clase (SDSS), como variables independientes. Para todos los análisis se utilizó el SPSS v.17.0.

\section{Resultados}

En primer lugar, se ofrecen los resultados descriptivos de las diferentes escalas estudiadas y a continuación las correlaciones bivariadas entre las mismas (Tabla 1). Respecto a las estrategias de disciplina, se obtuvo una alta puntuación media en el énfasis sobre razones intrínsecas para la disciplina, seguida de moderados valores en el énfasis sobre razones introyectadas y en la indiferencia del docente. En las dimensiones del clima motivacional, el clima al aprendizaje puntuó más alto que el clima al rendimiento. También la orientación a la tarea alcanzó valores superiores que la orientación al ego. En la escala de satisfacción, la satisfacción/diversión en las clases de EF duplicó la puntuación del aburrimiento.

Respecto a las correlaciones entre las dimensiones estudiadas, resalta la alta correlación positiva entre el énfasis sobre razones intrínsecas y el clima al aprendizaje, así como entre el énfasis sobre razones introyectadas y la indiferencia del docente. También es significativa la asociación entre clima al aprendizaje y clima al rendimiento, así como con la satisfacción/diversión en la EF. Resalta la alta correlación entre la orientación a la tarea y la orientación al ego. En la parte negativa, apuntar la correlación entre el aburrimiento y la satisfacción/diversión, el clima al aprendizaje y en énfasis sobre razones intrínsecas. 
Psicologia: Reflexão e Crítica, 28(2), 222-231.

Tabla 1

Media (M), desviación Típica (SD), y Correlaciones Bivariadas entre Variables

\begin{tabular}{lccccccccccc}
\hline \multicolumn{1}{c}{ Subescalas } & $M$ & $S D$ & 1 & 2 & 3 & 4 & 5 & 6 & 7 & 8 & 9 \\
\hline 1. Énfasis razones intrínsecas & 75.84 & 14.97 & $.05^{*}$ & $-.15^{* *}$ & $.58^{* *}$ & $.29^{* *}$ & $.18^{* *}$ & $.06^{* *}$ & $.41^{* *}$ & $-.19^{* *}$ \\
2. Énfasis razones introyectadas & 53.70 & 18.96 & & $.61^{* *}$ & -.01 & $.25^{* *}$ & $.08^{* *}$ & $.21^{* *}$ & $.07^{* *}$ & $.14^{* *}$ \\
3. Indiferencia del profesor & 48.73 & 17.53 & & & $-.17^{* *}$ & $.22^{* *}$ & -.01 & $.17^{* *}$ & -.01 & $.25^{* *}$ \\
4. Clima motivacional al aprendizaje & 68.86 & 17.98 & & & & $.45^{* *}$ & $.28^{* *}$ & $.11^{* *}$ & $.41^{* *}$ & $-.24^{* *}$ \\
5. Clima motivacional al rendimiento & 54.53 & 17.02 & & & & & $.26^{* *}$ & $.32^{* *}$ & $.22^{* *}$ & .01 \\
6. Orientación a la tarea & 3.91 & .69 & & & & & & $.76^{* *}$ & $.28^{* *}$ & $-.15^{* *}$ \\
7. Orientación al ego & 3.31 & 1.03 & & & & & & & $.16^{* *}$ & -.02 \\
8. Satisfacción/diversión & 4.16 & .87 & & & & & & & & $-.56^{* *}$ \\
9. Aburrimiento & 2.01 & 1.01 & & & & & & & & \\
\hline
\end{tabular}

${ }^{*} p<.05 ;{ }^{* *} p<.01$.

Todas las variables fueron estandarizadas en puntuaciones $Z$ y se procedió al agrupamiento del alumnado mediante análisis de conglomerados jerárquicos en el que se introdujeron las subescalas de los diferentes instrumentos: énfasis del profesor sobre razones intrínsecas para mantener la disciplina, énfasis del profesor sobre razones introyectadas para mantener la disciplina, indiferencia del profesor para mantener la disciplina, percepción de clima motivacional al aprendizaje, percepción de clima motivacional al rendimiento, orientación hacia la tarea, orientación hacia el ego, satisfacción/diversión y aburrimiento. De forma previa, se realizaron dos análisis de conglomerados, atendiendo las sugerencias de Hair et al. (1999). Se dividieron aleatoriamente los estudiantes por provincias andaluzas: el grupo A se compuso por el alumnado de las provincias de Granada y Sevilla; y el grupo B por el alumnado de las provincias de Almería, Córdoba y Jaén. Primero, y con el objetivo de identificar los perfiles motivacionales representados en el grupo A, se realizó un análisis jerárquico exploratorio con el método de Ward y en base a la lectura dendograma y a los resultados lógicos que se obtuvieron, se seleccionó una solución de tres conglomerados. Seguidamente se verificaron estos resultados obteniendo también tres perfiles motivacionales del grupo B mediante análisis de conglomerados a través del método $k$-medias. Por último se realizó un último análisis con toda la muestra a través del método de Ward. A la vista de los análisis previos (jerárquicos y no jerárquicos) y del dendograma que sugirió el análisis con el total de la muestra se adoptó la solución final de tres conglomerados (Tabla 2).

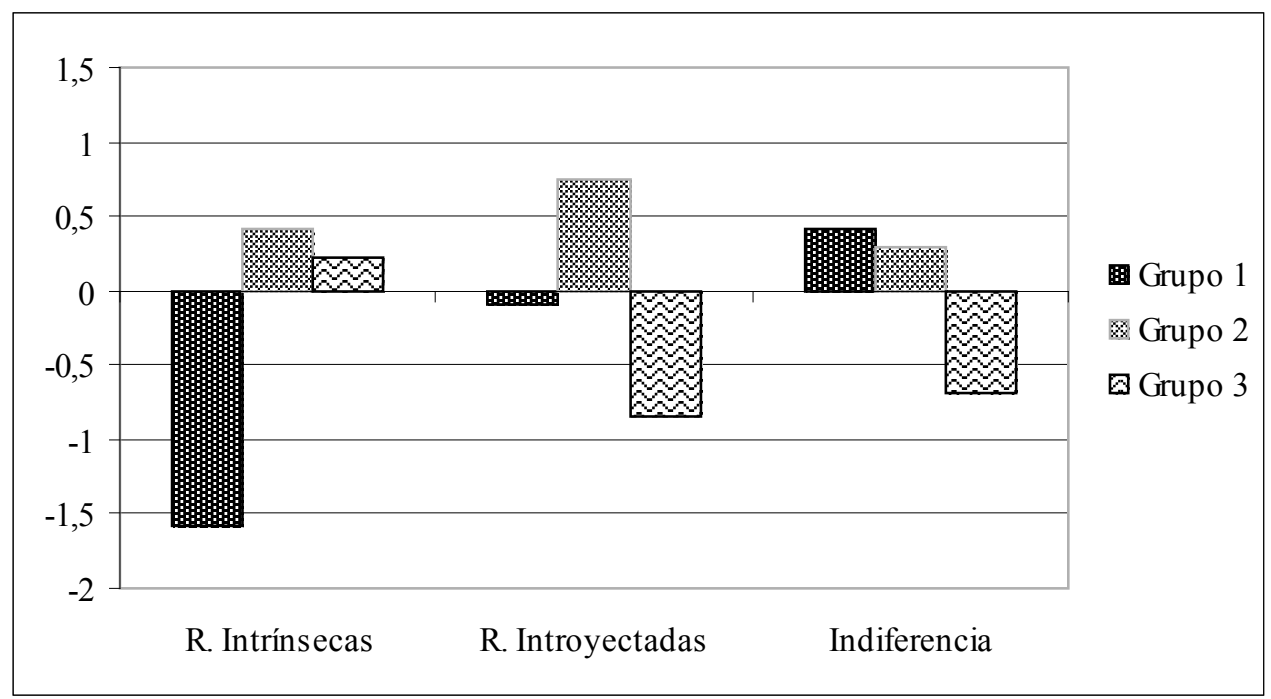

Figura 1. Peso de las variables de las estrategias para la disciplina utilizadas por el profesor en los tres conglomerados. En el eje vertical aparecen las puntuaciones $Z$. 
Se organizaron los conglomerados y se hallaron los grupos de alumnado que lo formaba en función de las subescalas de la Escala de Estrategias del Profesor para Mantener la Disciplina en Clase (SDSS). En la Figura 1, se observan los tres perfiles de estrategias para la disciplina por parte del docente con el análisis de conglomerados.

El conglomerado 1 se caracterizó por un perfil de indiferencia por parte del profesor en la disciplina y de muy bajo énfasis en razones intrínsecas. Es el conglomerado menos numeroso $(n=474)$, compuesto por un $54 \%$ de varones y un $46 \%$ de mujeres, mientras que el $65.4 \%$ practica ejercicio físico en su tiempo libre durante dos horas o más a la semana. En este perfil los valores más bajos correspondieron al clima motivacional al aprendizaje, orientación a la tarea y de satisfacción/diversión, y el valor más alto es el de aburrimiento.

El conglomerado 2 se caracterizó por un perfil de alto énfasis en razones introyectadas para la disciplina, con valores moderados de énfasis sobre razones intrínsecas y más bajos para la indiferencia por parte del docente. Está formado por un $54.8 \%$ de varones, un $45.2 \%$ de mujeres y por más de las dos terceras partes $(71 \%)$ de los que practican ejercicio físico más de dos horas a la semana. Este perfil se caracteriza por valores medios en las diferentes subescalas, correspondiendo los más positivos al clima motivacional al rendimiento, orientación al ego y satisfacción/diversión.

El alumnado clasificado en el grupo 3 formó el conglomerado más numeroso $(n=960)$ y tenían un perfil de énfasis sobre razones intrínsecas, con valores muy bajos en indiferencia y razones introyectadas. Este conglomerado está compuesto en su mayoría por mujeres, así como por dos terceras partes de quienes practican más de dos horas a la semana ejercicio físico (Tabla 2). Destacar que en este perfil se da el valor más alto de clima motivacional al aprendizaje y el más bajo de aburrimiento.

Tabla 2

Media (M), Desviación Típica (SD) y Puntuaciones Z para los Tres Conglomerados

\begin{tabular}{|c|c|c|c|c|c|c|c|c|c|}
\hline \multirow[b]{2}{*}{ Subescalas } & \multicolumn{3}{|c|}{ Grupo $1(n=474)$} & \multicolumn{3}{|c|}{ Grupo $2(n=568)$} & \multicolumn{3}{|c|}{ Grupo $3(n=960)$} \\
\hline & $M$ & $S D$ & $Z$ & $M$ & $S D$ & $Z$ & $M$ & $S D$ & $Z$ \\
\hline Énfasis razones intrínsecas & 52.17 & 12.40 & -1.58 & 82.04 & 10.46 & .41 & 77.63 & 11.29 & .22 \\
\hline Énfasis razones introyectadas & 55.54 & 16.52 & -.09 & 67.91 & 13.48 & .75 & 37.55 & 10.14 & -.85 \\
\hline Indiferencia del profesor & 55.89 & 14.17 & .41 & 57.27 & 16.98 & .29 & 36.84 & 11.28 & -.68 \\
\hline Clima motivacional al aprendizaje & 52.58 & 14.86 & -.90 & 72.48 & 16.72 & .20 & 70.94 & 17.14 & .12 \\
\hline Clima motivacional al rendimiento & 47.28 & 14.79 & -.42 & 60.27 & 16.82 & .34 & 51.26 & 16.12 & -.19 \\
\hline Orientación a la tarea & 3.73 & .76 & -.26 & 4.00 & .68 & .13 & 3.89 & .66 & -.04 \\
\hline Orientación al ego & 3.26 & 1.01 & -.05 & 3.49 & .99 & .17 & 3.13 & 1.04 & -.17 \\
\hline Satisfacción/diversión & 3.70 & .99 & -.52 & 4.33 & .75 & .20 & 4.13 & .89 & -.03 \\
\hline Aburrimiento & 2.23 & .97 & .22 & 2.08 & 1.09 & .07 & 1.84 & .90 & -.16 \\
\hline Características de los conglomerados & \multicolumn{3}{|c|}{$n(\%)$} & \multicolumn{3}{|c|}{$n(\%)$} & \multicolumn{3}{|c|}{$n(\%)$} \\
\hline Varones & \multicolumn{3}{|c|}{$256(54.0 \%)$} & \multicolumn{3}{|c|}{$311(54.8 \%)$} & \multicolumn{3}{|c|}{$378(39.4 \%)$} \\
\hline Mujeres & \multicolumn{3}{|c|}{$218(46.0 \%)$} & \multicolumn{3}{|c|}{$257(45.2 \%)$} & \multicolumn{3}{|c|}{$58260.6 \%)$} \\
\hline Practica $<$ de $2 \mathrm{~h} /$ semana ejercicio físico & \multicolumn{3}{|c|}{$164(34.6 \%)$} & \multicolumn{3}{|c|}{$165(29.0 \%)$} & \multicolumn{3}{|c|}{$341(35.5 \%)$} \\
\hline Practica 2 h o más/ semana ejercicio físico & \multicolumn{3}{|c|}{$310(65.4 \%)$} & \multicolumn{3}{|c|}{$403(71.0 \%)$} & \multicolumn{3}{|c|}{$619(64.5 \%)$} \\
\hline
\end{tabular}

Seguidamente se realizó un MANOVA con las dimensiones del clima motivacional, orientaciones de meta y satisfacción en las clases de EF como variables dependientes y los perfiles, según los grupos formados atendiendo a las subescalas del SDSS, como variables independientes. Emergió un efecto significativo multivariado, Lambda de Wilks $=.75, F(12,3990)=40.87, p<$ .001 . Los consiguientes análisis univariados expusieron diferencias significativas $(p<.001)$ en todas las variables.
En el contraste de comparaciones múltiples post hoc se realizó la prueba de Bonferroni. Estos análisis a posteriori (post hoc) comprueban las diferencias existentes entre todos los pares de medias.

Los valores de las diferentes dimensiones en cada conglomerado se pueden comprobar en la Tabla 2 y gráficamente en la Figura 2. En el clima motivacional al aprendizaje se hallaron diferencias significativas, $F(2$, $1999)=142.97, p<.001$, correspondiendo los valores más 
altos al grupo 2, seguidos del conglomerado $3 \mathrm{y}$, por último, el 1. Las pruebas post hoc (Bonferroni) expusieron que las diferencias entre el grupo 2 y el 1 fueron significativas $(p<.001)$, al igual que entre el 3 y el $1(p<.001)$. En el clima motivacional al rendimiento, $F(2,1999)=84.22, p$ $<.001$, el grupo 2 puntuó positivo, mientras que el 3 y el 1 lo hicieron de forma negativa. La prueba Bonferroni indicó diferencias significativas entre el conglomerado 2 y el 1 $(p<.001)$, entre el 2 y el $3(p<.001)$, así como entre el 3 y el $1(p=.002)$. También en la orientación a la tarea, $F$ $(2,1999)=17.47, p<.001$, la prueba de Bonferroni indicó diferencias entre los tres grupos, correspondiendo los valores más altos al grupo 2; destacan las diferencias entre el conglomerado 2 y el $1(p<.001)$. En la orientación al ego,
$F(2,1999)=24.15, p<.001$, también la puntuación más alta correspondió al grupo 2 , destacando las diferencias entre el conglomerado 2 y el 1 ( $p<.001$; Bonferroni). En la satisfacción intrínseca en la EF, el grupo 2 puntuó más alto en la dimensión de satisfacción/diversión, $F(2,1999)$ $=52.15, p<.001$, seguido del grupo $1 \mathrm{y}$, por último, del conglomerado 3. La prueba post hoc arrojó diferencias significativas $(p<.001)$ entre el grupo 2 y el 1 , entre el 2 y el 3, así como entre el 3 y el 1 . En el aburrimiento, $F$ $(2,1999)=17.84, p<.001$, el conglomerado 1 fue el que puntuó más alto, seguido del 2 y de 3 . La prueba de Bonferroni indicó que las principales diferencias $(p<.001)$ se producían entre el grupo 1 y 3 , así como entre el 2 y el 3; también entre el 1 y el 2 se hallaron diferencias $(p=.024)$.

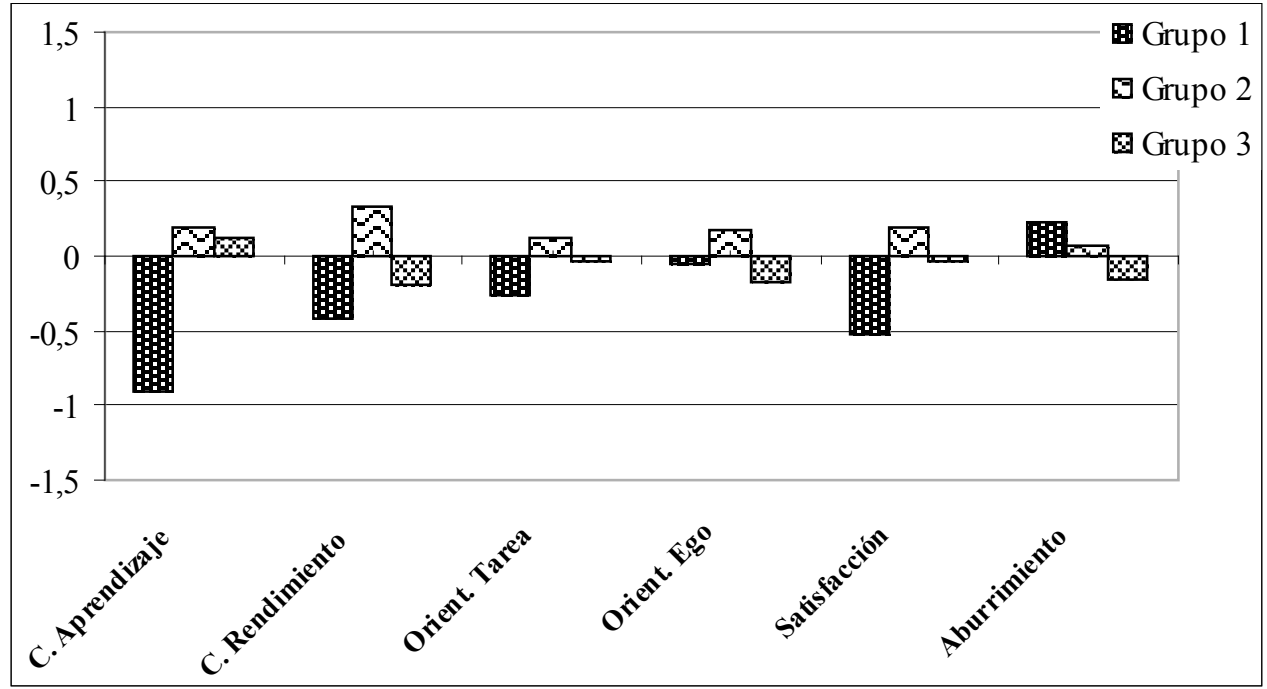

Figura 2. Peso del clima motivacional, orientaciones de meta y satisfacción en la EF en los tres conglomerados. En el eje vertical aparecen las puntuaciones $Z$.

\section{Discusión y Conclusiones}

El objetivo principal del estudio fue analizar las diferencias motivacionales de los alumnos en clase de EF según las estrategias de disciplina del docente. Para ello se llevó a cabo un análisis de conglomerados que ha demostrado la existencia de tres perfiles de disciplina. Un primer perfil con énfasis en estrategias autodeterminadas para mantener la disciplina en clase; un segundo, con énfasis en estrategias no autodeterminadas; $y$, un tercero, basado en la indiferencia del docente.

El primer objetivo secundario fue analizar las relaciones existentes entre los conglomerados y el clima motivacional percibido, las orientaciones de meta y la satisfacción con las clases de EF. Los resultados muestran que el perfil "indiferencia", se caracteriza por los valores más altos de aburrimiento y bajos de percepción de clima motivacional al aprendizaje, orientación a la tarea y satisfacción con las clases de EF. Estos resultados no coinciden con los hallados anteriormente por Moreno et al. (2008) y Papaioannou (1998), que confirmaron una relación positiva entre la orientación al ego y la regulación de la conducta mediante indiferencia, castigos y recompensas, es decir mediante razones introyectadas.

El segundo perfil se caracteriza por el "énfasis en razones introyectadas". Destaca un clima motivacional al rendimiento, orientación al ego y satisfacción con las clases de EF. Este segundo conglomerado ratifica los resultados expuestos por Spray (2002), a Cervelló et al. (2004), que demostraron que la percepción de un clima motivacional al rendimiento se relaciona positivamente con estrategias docentes basadas en razones externas. Asimismo, se ha demostrado que una orientación al ego acompañada de adecuadas sensaciones de competencia no son contraproducentes en un ambiente deportivo (Martínez-Galindo, Alonso, Cervelló, \& Moreno, 2009).

En el tercer perfil predomina el énfasis en razones intrínsecas. Se caracteriza por la percepción de un clima motivacional al aprendizaje, orientación a la tarea y los valores más bajos de aburrimiento. Tal como se demuestra, y coincidiendo con Moreno et al. (2008), existe una relación positiva entre la orientación a la tarea y el énfasis del do- 
cente en las razones intrínsecas para mantener la disciplina. Los resultados ratifican lo demostrado por Martínez-Galindo et al. (2009), Ntoumanis (2002), Wang y Biddle (2001), donde los perfiles motivacionales autodeterminados se relacionan con consecuencias motivacionales positivas como la diversión y la satisfacción. Asimismo, Martínez et al. (2005a) exponen que los alumnos motivados intrínsecamente no solo manifiestan gustarles y disfrutar con la $\mathrm{EF}$, sino que también perciben que su docente se preocupa por mantener el orden y la disciplina en clase. Por otro lado, Granero-Gallegos, Baena-Extremera, Gómez-López y Abraldes (2014) y Ntoumanis (2002) afirman que si un alumno se divierte tiende a estar intrínsecamente motivado y le concede mayor importancia a la asignatura de EF.

Por tanto, los resultados están en la línea de los hallados por Moreno et al. (2008) y Zounhia, Hatziharistos, y Emmanouel (2003), que demostraron que el perfil más autodeterminado se correspondía con los valores más altos de orientación a la tarea y estrategias de disciplina intrínsecas, frente al menos autodeterminado que correspondía a los valores más altos de orientación al ego y a estrategias de disciplina reguladas externamente. De este modo, se cumple casi en su totalidad la primera de las hipótesis planteadas, con la excepción de que en el segundo perfil es la diversión la que prevalece en vez del aburrimiento.

El segundo objetivo secundario fue hallar las diferencias de dichos conglomerados atendiendo al sexo y el $\mathrm{n}^{\circ} \mathrm{de}$ horas de ejercicio físico que realiza el alumno en su tiempo libre. Asimismo, para responder a la segunda de las hipótesis, el análisis de las diferencias entre los perfiles según el sexo ha demostrado que el conglomerado de estrategias autodeterminadas está formado sobre todo por mujeres, mientras que los conglomerados no autodeterminados lo forman sobre todo varones. Estos resultados están en la línea de los encontrados por Martínez, Alonso, Moreno y Cervelló (2005b), Moreno et al. (2006) y Moreno, Cervelló y Martínez (2007), que demostraron que las mujeres perciben que su docente se preocupa por mantener el orden y la disciplina en clase sobre todo por razones intrínsecas, mientras que los varones perciben que lo hace por razones introyectadas y de indiferencia.

Respecto al número de horas de ejercicio físico practicado a la semana en su tiempo libre, los resultados muestran que la gran mayoría de alumnos practican dos o más horas a la semana, siendo el conglomerado autodeterminado donde se encuentra el mayor porcentaje de practicantes que afirman hacerlo en esta cantidad de horas. En cambio, Moreno et al. (2005) reflejaron que los alumnos que practicaban deporte dos o tres días semanalmente percibían un mayor clima motivacional al rendimiento que los que practicaban deporte de manera puntual. Por otro lado, en el estudio reciente de GraneroGallegos et al. (2012) se mostró que aquellos alumnos que practicaban ejercicio físico más de tres horas a la semana se relacionaba con el perfil de alta motivación, caracterizado por estar los alumnos orientados al ego y a la tarea, sentir satisfacción con las clases y darle más importancia a la EF. Este último resultado coincide con el hallado por Moreno, Sánchez, Rodríguez, Prieto y Mula (2002), donde a medida que los estudiantes realizan más ejercicio físico en su tiempo libre mejorará la valoración de la asignatura. En cambio, en el estudio desarrollado por Martínez et al. (2005a) no se encontraron diferencias significativas entre las variables frecuencia de práctica deportiva fuera del horario escolar y motivación, razones de disciplina y percepción de las estrategias empleadas por los docentes para mantener el orden y la disciplina en clase. Por tanto, se cumple la segunda hipótesis, que hacía referencia a que los conglomerados basados en estrategias autodeterminadas estarían formados sobre todo por mujeres, mientras que los no autodeterminados lo estarían sobre todo por varones. Asimismo, también se hipotetizaba que los conglomerados basados en estrategias autodeterminadas estarían formados por los alumnos más activos en su tiempo libre, mientras que los conglomerados no autodeterminados lo estarían por los menos activos.

Finalmente, destacada la importancia del docente en las clases de EF (Granero-Gallegos \& Baena-Extremera, 2014), se ha demostrado que los docentes que quieran mejorar el ambiente de clase, favoreciendo un clima positivo de aprendizaje, deben potenciar el perfil de disciplina "razones intrínsecas" y controlar el perfil "razones introyectadas"; han de promover un clima motivacional al aprendizaje en las clases de EF que fomente una orientación a la tarea en los alumnos. Por otro lado, los resultados encontrados en el perfil "indiferencia" son bastantes preocupantes y han que provocar la reflexión de los docentes de EF de cara a intervenciones posteriores. En el trabajo desarrollado por Moreno y Cervelló (2010) se establecen propuestas de intervención para los docentes que les ayuden a solucionar los problemas de indisciplina en la clase de EF. Así, se debe crear un ambiente organizado, tranquilo y preocupado por las conductas de los estudiantes, establecer de forma positiva pocas normas en clase, describir el comportamiento de forma no juiciosa, expresar sus sentimientos y conocer los sentimientos de los alumnos, explicar los efectos que los comportamientos producidos tienen en ellos mismos y en los alumnos, establecer expectativas para futuros comportamientos, con el fin de reforzar el buen comportamiento y establecer consecuencias para el comportamiento indeseado y, por último, acabar la actuación docente de forma positiva.

Igualmente, los resultados han demostrado también que el docente deberá estar más atento a los varones y a aquel alumnado menos activos en su tiempo libre, ya que son los que representan el perfil menos autodeterminado y por tanto los que ostentan las conductas menos disciplinadas en las clases de EF.

Aunque el presente trabajo es una aproximación teórica cuyos resultados pueden ayudar a la elaboración de protocolos de actuación por parte de los docentes, hay que resaltar que presenta ciertas limitaciones derivadas del análisis de conglomerados. Es por ello, que los resultados deben ser considerados con precaución a causa del 
gran número de variables analizadas, que pueden influir en ellos (Boiché, Sarrazin, Grouzet, Pelletier, \& Chanal, 2008; Martínez-Galindo et al., 2009). Por ello, esperamos realizar en un futuro estudios de corte experimental que analicen el efecto que generan las diferentes intervenciones del docente en la mejora del clima de clase y la reducción de las conductas de indisciplina a través de la modificación intencionada de las estrategias de disciplina. De esta forma, los resultados contribuirán a diseñar intervenciones pedagógicas más focalizadas.

\section{Referencias}

Alonso, N., Martínez-Galindo, C., Moreno, J. A., \& Cervelló, E. (2005). Relación del género del alumno y el tipo de centro con la motivación, disciplina, trato de igualdad y estado de flow en Educación Física. In A. Díaz (Ed.), V Congreso Internacional de Educación Física e Interculturalidad. Murcia, España: Instituto de Ciencias del Deporte.

Baena-Extremera, A., Granero-Gallegos, A., Bracho-Amador, C., \& Pérez-Quero, F. J. (2012). Versión española del Sport Satisfaction Instrument (SSI) adaptado a la Educación Física. Revista de Psicodidáctica, 17, 377-396. doi:10.1387/Rev. Psicodidact.4496

Bekiari, A., Kokaridas, D., \& Sakellariou, K. (2006). Associations of students 'self-reports of their teachers' verbal aggression, intrinsic motivation, and perceptions of reasons for discipline in Greek physical education classes. Psychological Reports, 98, 451-461. doi:10.2466/pr0.98.2.451-461

Boiché, J., Sarrazin, P., Grouzet, F. M. E., Pelletier, L. G., \& Chanal, J. (2008). Students' motivational profiles and achievement outcomes in physical education: A self-determination perspective. Journal of Educational Psychology, 100, 688-701.

Cervelló, E., Jiménez, R., Del Villar, F., Ramos, I., \& SantosRosa, F. J. (2004). Goal orientations, motivational climate, equality, and discipline of Spanish physical education students. Perceptual and Motor Skills, 99, 271-283. doi:10.2466/ pms.99.1.271-283

Cervelló, E., Jiménez, R., Fenoll, A., Ramos, L., Del Villar, F., \& Santos-Rosa, F. J. (2002). A social-cognitive approach to the study of coeducation and discipline in Physical Education Classes. SOCIOTAM, Revista Internacional de Ciencias Sociales y Humanidades, 11, 43-64.

Deci, E. L., \& Ryan, R. M. (1985). Intrinsic motivation and self-determination in human behaviour. New York: Plenum.

Deci, E. L., \& Ryan, R. M. (2000). The "what" and "why" of goal pursuits: Human needs and the self-determination of behaviour. Psychological Inquiry, 11, 227-268.

Declaración de Helsinki. (2008). World Medical Association. Recuperado en http://www.wma.net/es/30publications/10policies/ b3/17c_es.pdf

Duda, J. L., \& Nicholls, J. G. (1992). Dimensions of achievement motivation in schoolwork and sport. Journal of Educational Psychology, 84, 290-299. doi:10.1037/0022-0663.84.3.290

Gómez-López, M., Granero-Gallegos, A., Baena-Extremera, A., \& Abraldes, J. A. (2013). Análisis de los perfiles motivacionales y su relación con la importancia de la educación física en secundaria. Revista Iberoamericana de Diagnóstico y Evaluación Psicológica, 36.

Gutiérrez, M., López, E., \& Ruiz, L. M. (2009). Estrategias para mantener la disciplina en las clases de educación física: va- lidación de su medida y análisis de la concordancia entre las percepciones de los profesores y las de sus alumnos. Revista Mexicana de Psicología, 26, 203-212.

Gutiérrez, M., Ruiz, L. M., \& López, E. (2010). Perceptions of motivational climate and teachers' strategies to sustain discipline as predictors of intrinsic motivation in physical education. Spanish Journal of Psychology, 13, 597-608. doi:10.1017/S1138741600002274

Granero-Gallegos, A., \& Baena-Extremera, A. (2014). Predicción de la motivación autodeterminada según las orientaciones de meta y el clima motivacional en Educación Física. Retos. Nuevas tendencias en Educación Física, Deporte y Recreación, 25, 23-27.

Granero-Gallegos, A., Baena-Extremera, A., Gómez-López, M., \& Abraldes, J. A. (2014). Estudio psicométrico y predicción de la importancia de la Educación Física a partir de las orientaciones de meta ("Perception of Success Questionnaire - POSQ”). Psicologia: Reflexão e Crítica, 27(3), 443-451. doi:10.1590/1678-7153.201427304

Granero-Gallegos, A., Baena-Extremera, A., Pérez-Quero, F. J., Ortiz-Camacho, M. M., \& Bracho-Amador, C. (2012). Analysis of motivational profiles of satisfaction and importance of physical education in high school adolescents. Journal of Sports Science and Medicine, 11, 614-623.

Hair, J. F., Anderson, R. E., Tatham, R. L., \& Black W. C. (1999). Análisis multivariante. Madrid, España: Prentice Hall Iberia.

Kulinna, P., Cothran, D., \& Regualos, R. (2003). Development of an instrument to measure student disruptive behavior. Measurement in Physical Education and Exercise Science, 7, 25-41. doi:10.1207/S15327841MPEE0701 3

Martínez, C., Alonso, N., \& Moreno, J. A. (2006). Análisis factorial confirmatorio del "Cuestionario de Percepción de Éxito (POSQ)" en alumnos adolescentes de Educación Física. In M. A. González, J. A. Sánchez, \& A. Areces (Eds.), IV Congreso de la Asociación Española de Ciencias del Deporte (pp. 757761). A Coruña, España: Xunta de Galicia.

Martínez, C., Alonso, N., Moreno, J. A., \& Cervelló. E. (2005a). Efectos de la práctica deportiva, la frecuencia de práctica y la satisfacción experimentada en la adopción de conductas disciplinadas en clases de educación física. In A. Díaz (Ed.), $V$ Congreso Internacional de Educación Física e Interculturalidad. Murcia, España: Instituto de Ciencias del Deporte.

Martínez, C., Alonso, N., Moreno, J. A., \& Cervelló. E. (2005b). La disciplina en educación física según el género del alumno y el tipo de centro. In A. Díaz (Ed.), V Congreso Internacional de Educación Física e Interculturalidad. Murcia, España: Instituto de Ciencias del Deporte.

Martínez-Galindo, C., Alonso, N., Cervelló, E., \& Moreno, J. A. (2009). Perfiles motivacionales y disciplina en clases de educación física. Diferencias según las razones del alumnado para ser disciplinado y la percepción del trato generado por el profesorado en el aula. Cultura y Educación, 21, 331-343. doi:10.1174/113564009789052361

Méndez-Giménez, A., Fernández-Río, J., Cecchini, J. A., \& González, C. (2013). Perfiles motivacionales y sus consecuencias en educación física. Un estudio complementario de metas de logro $2 \times 2$ y autodeterminación. Revista de Psicología del Deporte, 22, 29-38.

Moreno, J. A., Alonso, N., Martínez-Galindo, C., \& Cervelló, E. (2005). Motivación, disciplina, coeducación y estado de flow en educación física: diferencias según la satisfacción, la práctica deportiva y la frecuencia de práctica. Cuadernos de Psicología del Deporte, 5, 225-243. 
Moreno, J. A., \& Cervelló, E. (2010). Motivación en la actividad física y el deporte. Sevilla, España: Wanceulen Editorial Deportiva.

Moreno, J. A., Cervelló, E., \& Martínez, C. (2007). Perception of discipline according to gender, type of school, sport activity and interest in physical education in Spanish students. International Journal of Applied Sports Sciences, 19, 35-49.

Moreno, J. A., Conte, L., Hellín, P., Hellín, G., Vera, J. A., \& Cervelló, E. (2008). Predicción de la motivación autodeterminada según las estrategias para mantener la disciplina y la orientación motivacional en estudiantes adolescentes de Educación Física. Apuntes de Psicología, 26, 501-516.

Moreno, J. A., Hellín, P., Hellín, G., \& Cervelló, E. (2006). Efectos del género, la edad y la práctica físico-deportiva en las estrategias de disciplina, la orientación disposicional y la motivación autodeterminada en estudiantes adolescentes de Educación Física. In A. Díaz (Ed.), VI Congreso Internacional de Educación Física e Interculturalidad. Murcia, España: Instituto de Ciencias del Deporte.

Moreno, J. A., Sánchez, M., Rodríguez, D., Prieto, M. P., \& Mula, C. (2002). Valoración de la educación física según la importancia concedida por el alumno. In A. Díaz, J. A. Moreno, \& P. L. Rodríguez (Eds.), Congreso de Educación Física e Interculturalidad. Murcia, España: Consejería de Educación.

Nicholls. J. G. (1989). The competitive ethos and democratic education. Cambridge, MA: Harvard University Press.

Ntoumanis, N. (2002). Motivational clusters in a sample of British physical education classes. Psychology of Sport and Exercise, 3, 177-194.

Papaioannou, A. (1998). Goal perspectives, reasons for being disciplined, and self-reported discipline in physical education lessons. Journal of Teaching in Physical Education, 17, 421-441.

Roberts, G. C., Treasure, D. C., \& Balagué, G. (1998). Achievement goals in sport: The development and validation of the Perception of Success Questionnaire. Journal of Sport Sciences, 16, 337-347. doi:10.1080/02640419808559362

Rutten, C., Boen, F., \& Seghers, J. (2012). How school social and physical environments relate to autonomous motivation in physical education: The mediating role of need satisfaction. Journal of Teaching in Physical Education, 31, 216-230.

Ryan, R. M., \& Deci, E. L. (2000). Self-determination theory and the facilitation of intrinsic motivation. social development and well-being. American Psychologist, 55, 68-78.

Spray, C. M. (2002). Motivational climate and perceived strategies to sustain pupils' discipline in physical education. European Physical Education Review, 8, 5-20. doi:10.1177/1356336X020081001

Spray, C. M., \& Wang, C. K. J. (2001). Goal orientations, self-determination and pupils' discipline in physical education. Journal of Sports Sciences, 19, 903-913. doi:10.1080/026404101317108417

Wang, J., \& Biddle, S. (2001). Young people's motivational profiles in physical activity: A cluster analysis. Journal of Sport and Exercise Psychology, 23, 1-22.

Zounhia, K., Hatziharistos, D., \& Emmanouel, K. (2003). Greek secondary schools pupils' perceived reasons for behaving appropriately and perceived teachers' strategies to maintain discipline. Educational Review, 55, 289-303. doi:10.1080/0013191032000118947 